\title{
An improved method for extracting matrix elements from lattice three-point functions
}

\section{Aubin*}

Department of Physics, Fordham University, Bronx, NY, USA

E-mail: caubinefordham.edu

\section{K. Orginos}

Department of Physics, College of William and Mary, Williamsburg, VA, USA

Jefferson Laboratory, Newport News, VA, USA

The extraction of matrix elements from baryon three-point functions is complicated by the fact that the signal-to-noise drops rapidly as a function of time. Using a previously discussed method to improve the signal-to-noise for lattice two-point functions, we use this technique to do so for lattice three-point functions, using electromagnetic form factors for the Delta as an example.

XXIX International Symposium on Lattice Field Theory

July 10 - 162011

Squaw Valley, Lake Tahoe, California

${ }^{*}$ Speaker. 


\section{Introduction}

The calculation of the electromagnetic form factors for mesons and baryons is crucial to understanding the structure of hadronic states in QCD. However, for the $\Delta(1232)$ and other extremely short-lived resonances, they are notoriously difficult to both measure experimentally as well as calculate theoretically due to the complications that arise from the strong interactions. In the case of the $\Delta(1232)$ resonance, the form factors themselves are not currently experimentally accessible, although two of the form factors in the static limit are known (the charge) or measured to some degree (the magnetic dipole moment). For the nucleon, experimental results do exist for the form factors as a function of the momentum transfer. This makes a calculation of the nucleon form factors both a check of methodology as well as of QCD, and allows us to be confident that lattice results for the $\Delta$ form factors are reasonable. Such an analysis will occur in a forthcoming publication [1].

The electromagnetic form factors of the $\Delta$ are encoded in the matrix element

$$
\left\langle\Delta\left(p^{\prime}\right)\left|J_{\mu}\right| \Delta(p)\right\rangle=\bar{u}_{\alpha}\left(p^{\prime}\right) \Gamma_{\alpha \beta \mu} u_{\beta}(p)
$$

where $u_{\alpha}$ is a Rarita-Schwinger vector-spinor describing the external $\Delta$, and $J_{\mu}=\sum_{q} \bar{q} \gamma_{\mu} q$ is the electromagnetic vector current. The Lorentz structure of $\Gamma$ is given, for example, in [2], and has four form factors $F_{1,2,3,4}^{*}\left(Q^{2}\right)$ that are functions of $Q^{2}=-\left(p^{\prime}-p\right)^{2}$ alone. These form factors give rise, in the limit $Q^{2} \rightarrow 0$, to the electric charge, magnetic dipole moment, electric quadrupole moment, and magnetic octupole moment of the $\Delta$.

Of these moments, the charge is of course known, and from the PDG [3], we have

$$
\begin{aligned}
\mu_{\Delta^{++}} & =(5.6 \pm 1.9) \mu_{N}, \\
\mu_{\Delta^{+}} & =(2.7 \pm 3.5) \mu_{N},
\end{aligned}
$$

where we have added all of the errors (including theoretical) in quadrature just to get an idea for how well these are determined experimentally. Thus, it is essential even for this simple quantity to have a well-determined lattice result. Some unquenched results were obtained using the form factor approach in [4] and using a background field technique [5], but there are several difficulties that arise in these different methods. For this work, we will focus on the difficulties with the form factor approach.

\section{Lattice three-point functions}

These are determined by calculating the 3-point correlator:

$$
C^{3 \mathrm{pt}}\left(t_{i}, t, t_{f}, \mathbf{p}_{i}, \mathbf{p}_{f}\right)=F T\left[\left\langle 0\left|\chi\left(t_{f}, \mathbf{x}_{f}\right) J_{\mu}(t, \mathbf{0}) \bar{\chi}\left(t_{i}, \mathbf{x}_{i}\right)\right| 0\right\rangle\right],
$$

where $F T$ is the Fourier Transform of the correlator, and $\chi$ is some appropriate interpolating operator for the $\Delta$. In the large time limit $t_{f} \gg t \gg t_{i}$, for example, if we are interested in determining $G_{E 0}$, we can set $\mu=4$ and we get, using the $+3 / 2$ spin projection for both of the external $\Delta$ 's,

$$
C_{s s}^{3 \mathrm{pt}}\left(t_{i}, t, t_{f}, \mathbf{p}_{i}, \mathbf{p}_{f}\right) \rightarrow \frac{C_{\mathrm{sp}}^{2 \mathrm{pt}}\left(t, \mathbf{p}_{i}\right)}{C_{\mathrm{SS}}^{2 \mathrm{pt}}\left(t_{f}-t_{i}, \mathbf{p}_{f}\right) C_{\mathrm{sp}}^{2 \mathrm{pt}}\left(t, \mathbf{p}_{f}\right)}\left\langle\Delta\left(p_{f},+3 / 2\right)\left|J_{4}(0)\right| \Delta\left(p_{i},+3 / 2\right)\right\rangle+\cdots,
$$



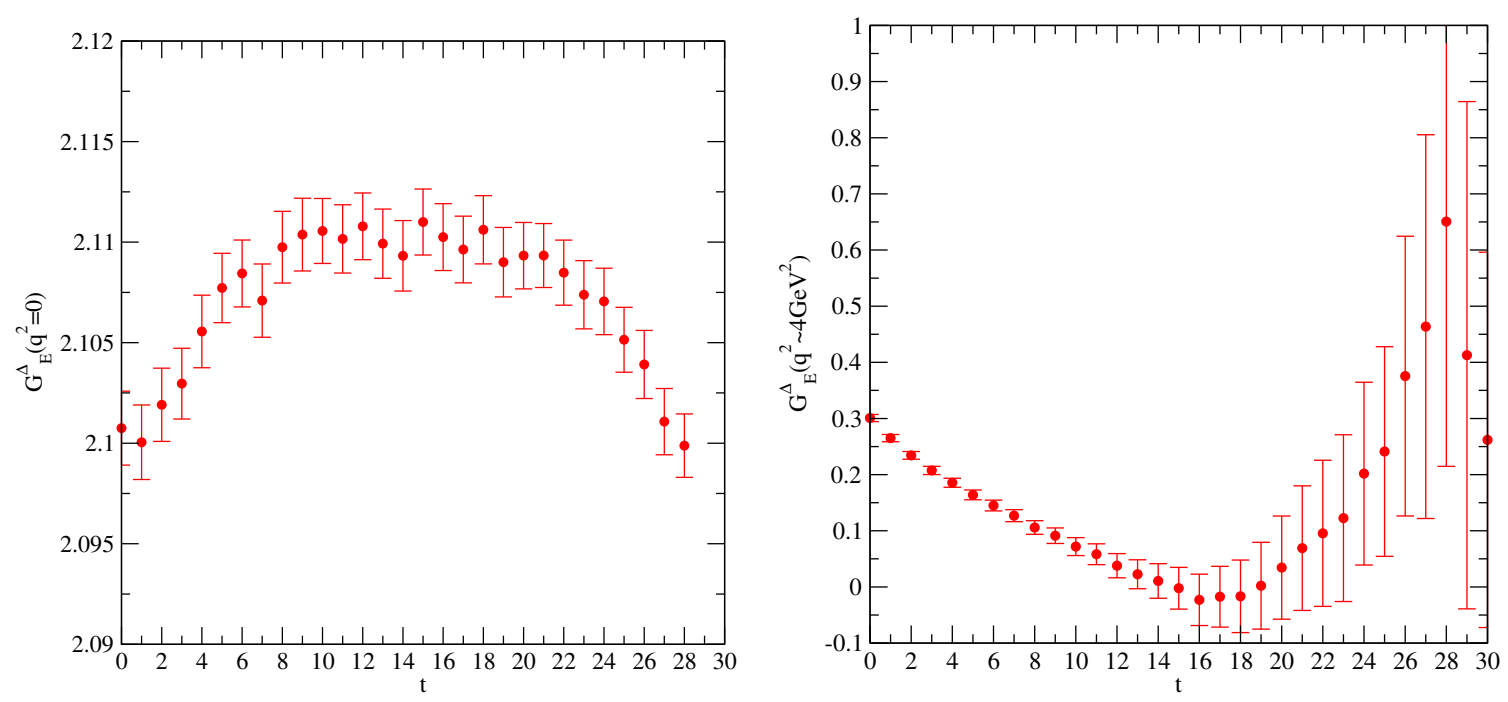

Figure 1: On the left is the matrix element $G_{E}$ for the $\Delta^{++}$evaluated at $q^{2}=0$. While there is significant excited state contamination, there is a noticeable plateau that gives (up to renormalization) the charge of the $\Delta$. On the right is the same matrix element for $q^{2}=11\left(2 \pi / a_{s} L\right)^{2} \approx 4 \mathrm{GeV}^{2}$, where there is no trustworthy signal.

with

$$
\left\langle\Delta\left(p_{f},+3 / 2\right)\left|J_{4}(0)\right| \Delta\left(p_{i},+3 / 2\right)\right\rangle=G_{E 0}\left(q^{2}\right),
$$

and $C_{\mathrm{ss}}^{2 \mathrm{pt}}$ and $C_{\mathrm{sp}}^{2 \mathrm{pt}}$ are the shell-shell and shell-point two-point functions. The dots denote contributions from excited states that are generally ignored. Note that additional complications arise in separating the $\Delta$ state and the $N-\pi$ state when the pion mass is below $300 \mathrm{MeV}$. Currently we are not near this regime, so it is not an issue for the current analysis.

The problem is that the contamination from excited states can be seen to be large, even for the simplest cases. Take here the $\Delta^{++} E 0$ form factor, $G_{E 0}\left(q^{2}\right)$, which is a linear combination of the $F_{i}^{*}$ form factors. In the limit $q^{2} \rightarrow 0, G_{E 0}(0)=+2$, the electric charge of the $\Delta^{++}$in units of $|e|$. We have shown in Ref. [6] preliminary results from analyzing these data via the standard technique, even though there was significant contamination from excited states.

We show on the left of Fig. 1 the extraction of $G_{E 0}$ for two values of momenta using the Hadron Spectrum collaboration lattices of 2+1-flavor anisotropic Clover lattices [7] (here with a volume of $16^{3} \times 128$ and a pion mass of roughly $490 \mathrm{MeV}$, and $a_{t}^{-1} \approx 5.5 \mathrm{GeV}, a_{s} / a_{t} \approx 3.5$ ). The source and sink are located at $t=0,28$, and we can see a plateau in the center where we could reliably extract the form factor, but there is significant contamination from excited states, seen from the deviation from the plateau.

\section{Generalized Eigenvalue Problem}

As discussed in Ref. [6], we can use a modification of the Generalized Eigenvalue Problem (GEVP), noting that there are many interpolating operators that could be used to calculate a twopoint function, and one could form a matrix of correlators

$$
C_{i j}^{2 \mathrm{pt}}(t)=\left\langle 0\left|O_{i}(t) \bar{O}_{j}(0)\right| 0\right\rangle .
$$


From this, by solving the GEVP

$$
C^{2 \mathrm{pt}}(t) \mathbf{v}_{n}=\lambda_{n}\left(t, t_{0}\right) C^{2 \mathrm{pt}}\left(t_{0}\right) \mathbf{v}_{n}
$$

one can show (see [8] and references therein) that the eigenvalues behave like

$$
\lambda_{i}\left(t, t_{0}\right) \sim e^{-m_{i}\left(t-t_{0}\right)}+\cdots .
$$

The reference time $t_{0}$ is empirically chosen so that at that time, all $n$ states (for an $n \times n$ system) would contribute to the correlator; no more, no less. For our purposes, we find that the choice of $t_{0}$ has little effect on our results, primarily because we are using a very small basis of operators.

\section{Generalized Pencil-of-Function Method}

While the GEVP is very useful for obtaining reliable extractions of excited states, one can use a different approach that can kill off the excited state contribution to the ground state. This is known as the Generalized Pencil-of-Function (GPoF) method $[9,10]$. In a quantum mechanical system, the important point is that if $O_{\Delta}(t)$ is an interpolating operator for the $\Delta$, then so is

$$
O_{\Delta}^{\tau}(t) \equiv e^{H \tau} O_{\Delta}(t) e^{-H \tau}=O_{\Delta}(t+\tau),
$$

and this new operator is linearly independent from the original operator.

So if we use $O_{\Delta}(t), O_{\Delta}^{\tau}(t)$ as our two operators, we can construct a correlator matrix using only a single correlator. This matrix is

$$
\mathbf{C}(t)=\left(\begin{array}{cc}
C(t) & C(t+\tau) \\
C(t+\tau) & C(t+2 \tau)
\end{array}\right) .
$$

We can replicate this and use a set of operators $O_{\Delta}^{\tau, n}(t)=O_{\Delta}(t+n \tau)$, and make this correlator as large as we wish. It turns out that using multiple shifts does not give us any additional information, so we will ultimately use just a single shift and a $\tau=4$. This would be something that is determined for each correlator one is interested in. Once this matrix is created then we follow through the same procedure as before with the GEVP. More details can be found in $[6,11]$

When applying this to three-point correlator data, we first solve the GEVP for the shifted two-point correlator, by first performing a singular-value decomposition on $C^{2 \mathrm{pt}}\left(t_{0}\right)$, so

$$
C^{2 \mathrm{pt}}\left(t_{0}\right)=U \Sigma V^{\dagger},
$$

where $\Sigma$ has a dimension $\leq C^{2 \mathrm{pt}}\left(t_{0}\right)$, where we remove singular values that are smaller than the largest singular value by some cutoff, say $10^{-3}$. Then we solve

$$
\bar{C}^{2 \mathrm{pt}}(t) \mathbf{v}_{n}^{\prime}=\lambda_{n}\left(t, t_{0}\right) \mathbf{v}_{n}^{\prime},
$$

with

$$
\bar{C}^{2 \mathrm{pt}}(t)=\Sigma^{-1 / 2} U^{\dagger} C^{2 \mathrm{pt}}(t) V \Sigma^{-1 / 2}, \quad \mathbf{v}_{n}^{\prime}=\Sigma^{1 / 2} V^{\dagger} \mathbf{v}_{n},
$$

and then we use these solutions to diagonalize the three-point correlator, so that

$$
\bar{G}_{n n^{\prime}}^{3 \mathrm{pt}}=\left(\mathbf{v}^{T \prime} \Sigma^{-1 / 2} U^{\dagger}\right)_{n i} G_{i j}^{3 \mathrm{pt}}\left(V \Sigma^{-1 / 2} \mathbf{v}^{\prime}\right)_{j n^{\prime}} .
$$

We then use this version of the three-point function as well as the "barred" two-point functions [Eq. (4.4)] in Eq. (2.2) to extract the form factor $G_{E 0}$. 

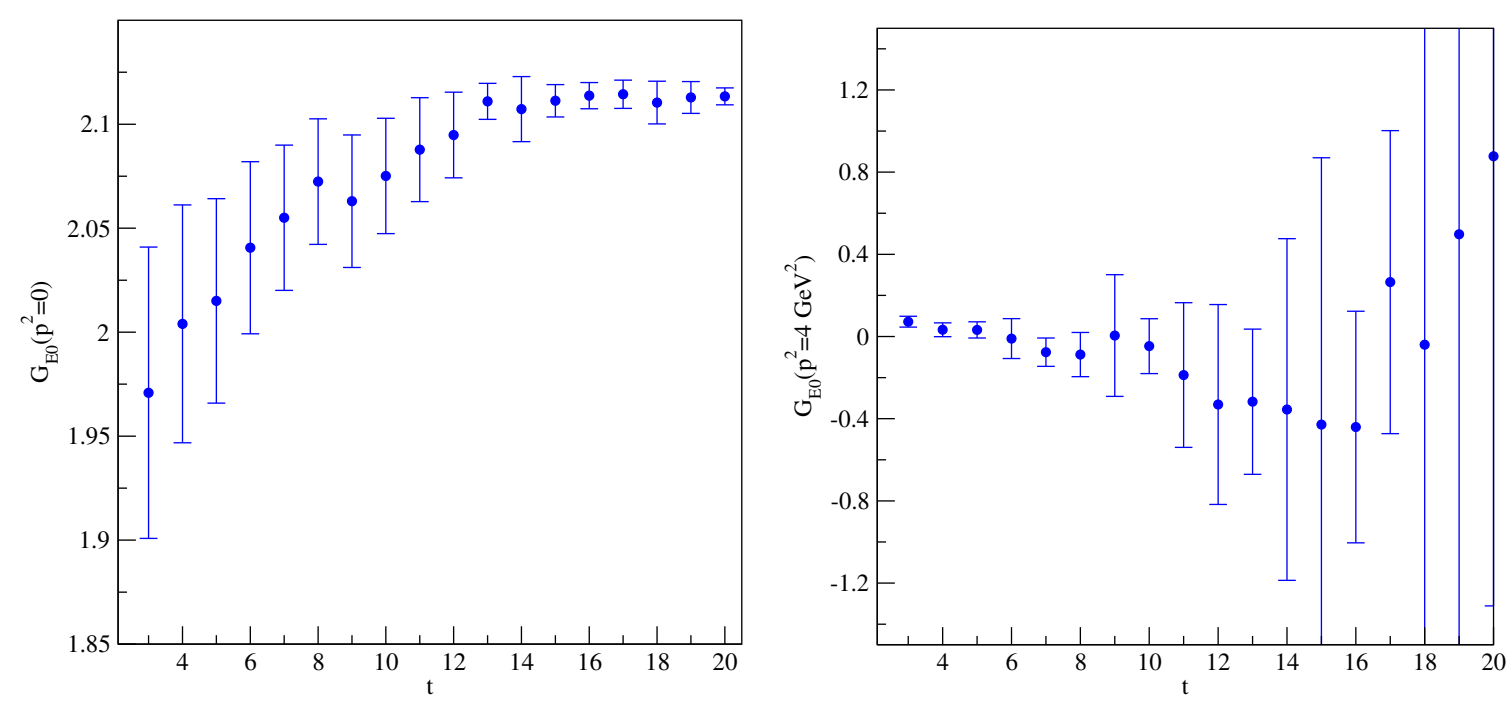

Figure 2: The same as Fig. 1, but for the three-point functions analyzed by the GPoF method.

\section{Preliminary Results}

We show initial results for this approach for the two momenta chosen above in Fig. 2. Here we use three source-sink separations of 20,24, and 28. We see that the GPoF approach has improved the plateaux noticeably. There is still excited-state contamination for the zero-momentum contribution, but the resulting plateau has smaller statistical errors. As for the higher momentum plot $p^{2} \approx 4$ $\mathrm{GeV}^{2}$, the overall result is consistent with zero, and the GPoF approach significantly improves the signal.

We show in Fig. 3 results from extracting the form factor $G_{E 0}$ (divided by 2 to compare with that of the proton as seen in Ref. [6]) as a function of $p^{2}$, the momentum transfer. While a more detailed analysis of the systematics needs to be performed (along the lines of that in Ref. [11]), these results show that with a small amount of additional computation, one can extract reliable plateaux from lattice three-point functions with the excited-state contamination significantly reduced.

\section{References}

[1] C. Aubin and K. Orginos, in preparation.

[2] V. Pascalutsa, M. Vanderhaeghen, and S. N. Yang, Electromagnetic excitation of the $\Delta(1232)$ resonance, Phys. Rept. 437 (2007) 125-232.

[3] Particle Data Group Collaboration, K. Nakamura, Review of particle physics, J. Phys. G37 (2010) 075021.

[4] C. Alexandrou et. al., Delta-baryon electromagnetic form factors in lattice QCD, Phys. Rev. D79 (2009) 014507, [arXiv: 0810.3976].

[5] C. Aubin, K. Orginos, V. Pascalutsa, and M. Vanderhaeghen, Magnetic Moments of $\Delta$ and $\Omega^{-}$ Baryons with Dynamical Clover Fermions, Phys. Rev. D79 (2009) 051502.

[6] C. Aubin and K. Orginos, A new approach for Delta form factors, AIP Conf.Proc. 1374 (2011) 621-624, [arXiv: 1010.0202]. 


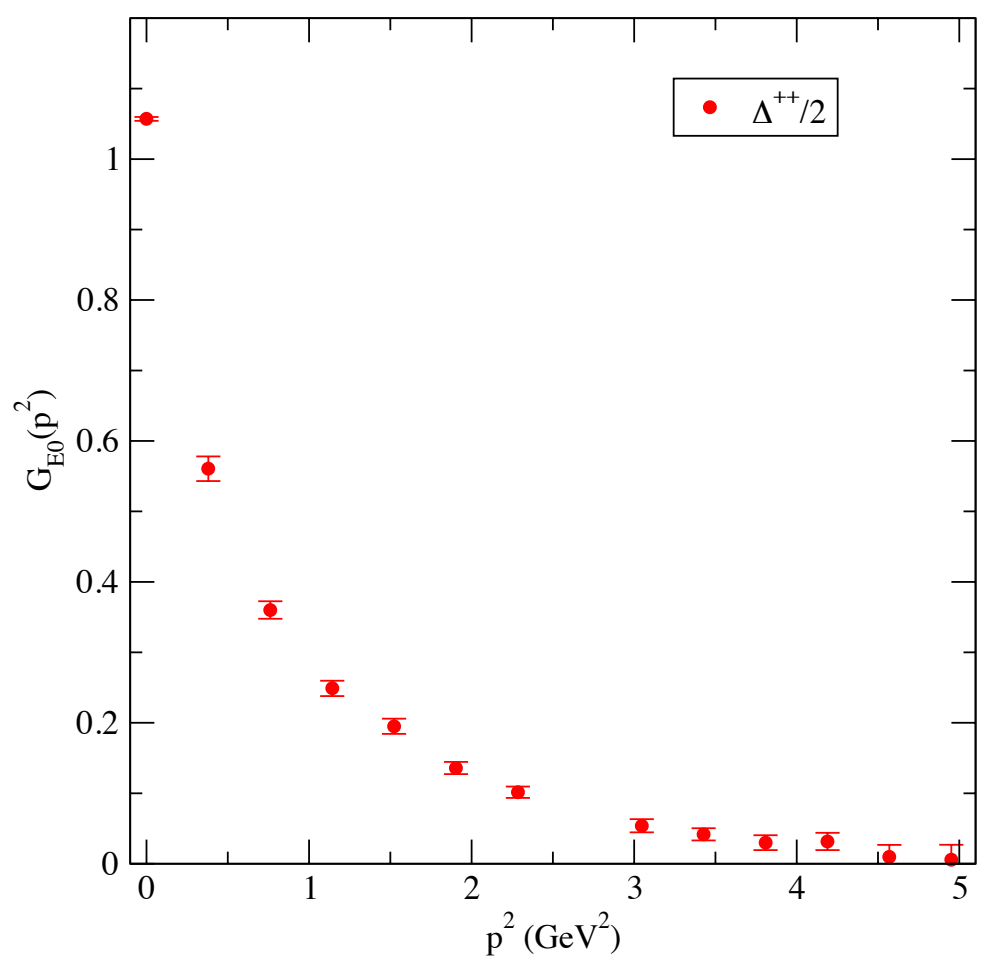

Figure 3: Preliminary extraction of the $G_{E 0}$ form factor using the GPoF method.

[7] Hadron Spectrum Collaboration, H.-W. Lin et. al., First results from 2+1 dynamical quark flavors on an anisotropic lattice: light-hadron spectroscopy and setting the strange-quark mass, Phys. Rev. D79 (2009) 034502.

[8] B. Blossier, M. Della Morte, G. von Hippel, T. Mendes, and R. Sommer, On the generalized eigenvalue method for energies and matrix elements in lattice field theory, JHEP 04 (2009) 094.

[9] Y. Hua and T. Sarkar, Generalized pencil-of-function method for extracting poles of an em system from its transient response, IEEE transactions on antennas and propagation 37 (1989) 229-234.

[10] T. Sarkar and O. Pereira, Using the matrix pencil method to estimate the parameters of a sum of complex exponentials, IEEE Antennas and Propagation Magazine 37 (1995) 48-55.

[11] C. Aubin, K. Orginos, and A. Walker-Loud, Generalized pencil-of-function method for analyzing lattice data, in preparation. 